\title{
AVIS AUX LECTEURS
}

Le Comité de rédaction de Modèles linguistiques

\section{(2) OpenEdition}

Journals

Édition électronique

URL : https://journals.openedition.org/ml/564

DOI : $10.4000 / \mathrm{ml} .564$

ISSN : 2274-0511

\section{Éditeur}

Association Modèles linguistiques

\section{Édition imprimée}

Date de publication : 1 juillet 2006

Pagination : 5-7

\section{Référence électronique}

Le Comité de rédaction de Modèles linguistiques, "AVIS AUX LECTEURS », Modèles linguistiques [En ligne], 54 | 2006, mis en ligne le 01 octobre 2015, consulté le 01 juillet 2021. URL : http:// journals.openedition.org/ml/564; DOl : https://doi.org/10.4000/ml.564

Ce document a été généré automatiquement le 1 juillet 2021.

(c) Modèles Linguistiques 


\title{
AVIS AUX LECTEURS
}

\author{
Le Comité de rédaction de Modèles linguistiques
}

1 Le présent volume de Modèles linguistiques constitue le second volet d'un triptyque consacré à la préposition en français. On trouvera le premier volet dans le volume 54 de la revue (tome XXVII-1, 2006, pp. 7-144). Le troisième, plus particulièrement centré sur les locutions prépositionnelles, paraîtra en 2007 (tome XXVIII-1). Trois numéros sur le même thème: cela ne s'était pas vu dans notre revue depuis 1994-95 ( Temps et langage », vol. 31, 32, 33).

2 Il s'agit donc là, sur un thème forcément plus réduit (une seule catégorie grammaticale, une seule langue) d'une entreprise exceptionnelle dont l'organisation a été exceptionnellement confiée à Danielle Leeman, professeur à l'Université de Paris XNanterre et directrice de l'UMR 7114 (MoDyCo), bien connue par ailleurs pour ses activités éditoriales à Langage et à Langue française. Lui confier la responsabilité du contenu de ce numéro et de son économie s'imposait d'autant plus qu'un certain nombre de participants sont membres de l'équipe qu'elle dirige. On a donc là, d'une certaine manière, les résultats d'un travail de recherche collectif qui a donné lieu à de nombreuses discussions dans des séminaires réguliers. Quant aux autres collaborateurs extérieurs à l'équipe, ils ont été réunis, avec les membres "ordinaires", si l'on peut dire, dans des mini-colloques, afin de mettre au point et d'harmoniser les diverses contributions. C'est dire le soin et le sérieux avec lequel D. Leeman a préparé ces trois volumes. Qu'elle en soit remerciée.

3 L'intervention de Modèles linguistiques s'est limitée au plan éditorial, ce qui n'a pas été sans nous poser quelques problèmes. Ainsi, le présent numéro, sur un seul thème, est volumineux par rapport aux normes ordinaires. Il a fallu, ici et là, mais le plus rarement possible, réduire certaines notes au strict nécessaire pour une bonne compréhension du corps du texte. Un article de revue n'est pas exactement un exposé de séminaire, qui peut comporter digressions et apartés : pour d'évidentes raisons budgétaires, il a fallu se plier aux lois du genre.

4 Afin de répondre aux souhaits de la responsable, la revue a accepté, quelque peu inconsidérément, il faut bien le dire, de publier une «Bibliographie générale ». L'idée est en soit excellente. Les problèmes se sont présentés lorsqu'il s'est avéré que cette 
bibliographie faisait dans les 70 pages, soit près de la moitié d'un numéro normal de la revue... La solution envisagée, elle aussi excellente, a été de la publier sous la forme d'un fascicule séparé. Pour différentes raisons, elle n'a pu être réalisée.

5 La solution que nous avons trouvée, et dont nous assumons la pleine responsabilité, a été de partager cette bibliographie générale en deux : une partie dans le volume 54 , ici même, la seconde dans le volume 55, à paraître. De plus, comme nombre d'articles, dans leurs "références bibliographiques ", renvoient forcément aux mêmes sources et aux mêmes auteurs, nous avons décidé de tout regrouper à la fin du volume, pratique courante dans les deux revues mentionnées ci-dessus. Les lecteurs ne devraient pas s'en trouver trop gênés.

6 La bibliographie linguistique de ces trente dernières années, pour ne pas remonter plus haut, fait apparaître qu'il y a périodiquement des thèmes privilégiés (cf. ouvrages, actes de colloques, articles de revues). Il appartiendra à l'historien du futur de découvrir pourquoi tel ou tel sujet est choisi de préférence à tel autre et d'essayer de déterminer comment naît une mode. La préposition est un de ces thèmes actuellement «à la mode ». Ainsi, quelques mois après la parution du présent volume, et pratiquement en même temps que la parution du volume 55, prévue en septembre prochain, on annonce un colloque intitulé «Autour de la préposition (position, valeurs, statut et catégories apparentées à travers les langues) $\aleph^{\mathrm{i}}$. Par son ouverture sur d'autres langues, ce colloque ne fait pas double emploi avec les articles publiés dans Modèles linguistiques. Il en est complémentaire, ce dont nous nous réjouissons à l'avance.

7 Dans l'effervescence actuelle autour du thème prépositionnel, notre revue tient à rappeler et à réactualiser son objectif premier, tel qu'il était formulé dans le numéro d'ouverture, à savoir "susciter la discussion $»^{\mathrm{ii}}$. Modèles linguistiques a publié par le passé des commentaires (articles critiques, comptes rendus) sur des articles parus dans des numéros précédents, mais il ne s'agissait pas à proprement parler de « discussion ». Or la préposition se prête tout particulièrement à la discussion. La lecture des articles des volumes 53 et 54 (et, prévisionnellement, sans doute celle du vol. 55) montre en effet que la préposition est une catégorie difficile à cerner, à décrire dans ses emplois et son fonctionnement, à théoriser. Peut-on parler à son propos de «modèles »? Si oui, quels sont ces modèles? La plupart des articles reflètent ce qu'on appelle en anglais "work in progress». C'est avec prudence que les auteurs proposent une analyse, avancent une explication, esquissent une hypothèse. Nous ne nous en plaindrons pas. Mais on peut souhaiter davantage. De plus, ces analyses, ces explications, ces hypothèses sont-elles toujours pertinentes? Autant de questions qui peuvent être posées dans un colloque et auxquelles les auteurs peuvent essayer de répondre. Il est malheureusement très rare que questions et réponses soient publiées dans les actes de colloques.

8 C'est pourquoi nous proposons à nos lecteurs un débat autour de la préposition. Ce débat prendrait la forme de questions à propos d'un ou de plusieurs articles, ou de l'ensemble d'un volume. Nous disons bien "questions»; il ne s'agit pas de proposer une analyse, mais tout au plus de suggérer des réponses possibles (p. ex. « Ne pensezvous pas que.?»). Ces réponses seraient apportées par les auteurs eux-mêmes, s'ils acceptent de "jouer le jeu». Les lecteurs intéressés - nous espérons qu'ils seront nombreux - peuvent donc adresser leur(s) question(s) à l'adresse électronique de la revue: revuemodling@wanadoo.fr Nous transmettrons aux auteurs. Questions et réponses seront publiées. C'est ainsi, pensons-nous, qu'avance la recherche. 
Il nous reste à remercier le Conseil scientifique de l'Université de Paris X-Nanterre, par l'intermédiaire de l'UMR 7114, de nous avoir accordé une subvention qui permettra de financer une bonne partie du prochain numéro (vol. 55). Que ses membres en soient vivement remerciés. Le présent numéro est financé pour moitié par l'équipe BABEL de l'Université du Sud, Toulon-Var. Il va sans dire que nous lui adressons aussi tous nos remerciements.

\section{NOTES DE FIN}

i. Ce colloque est organisé par le CRISCO, Université de Caen, les 20, 21 et 22 septembre 2007. Pour tous renseignements complémentaires (programme, inscriptions) consulter le site http:// www.crisco.unicaen.fr

ii. [...] si notre revue peut favoriser la critique interne et externe des modèles, leur élaboration et leur application à des problèmes du langage de toute nature, non seulement par des linguistes, mais dans un cadre de discussion interdisciplinaire, par des logiciens, des philosophes, des informaticiens, des psycholinguistes, alors notre but sera pleinement atteint (Modèles linguistiques, tome I, fasc.1, 1979). 